
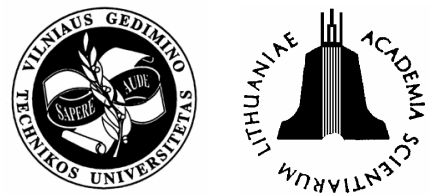

\title{
SIMULATION-BASED FORECASTING EFFECTS OF AN ACCIDENTAL EXPLOSION ON THE ROAD. PART II: CASE STUDY
}

\author{
Egidijus R. Vaidogas \\ Dept of Reinforced Concrete Structures, Vilnius Gediminas Technical University, \\ Sauletekio al. 11, LT-10223 Vilnius, Lithuania.E-mail: erv@st.vtu.lt
}

Received 19 April 2006; accepted 4 September 2006

\begin{abstract}
The paper contains a case study covering forecasting mechanical effects of an explosion which can be generated during a road accident. It illustrates a practical application of the simulation-based procedure developed for such forecasting in the first part of the paper. The case study reveals the amount and character of the knowledge necessary to carry out this forecasting. Its final result is a probabilistic model describing likelihood of occurrence of accidental explosion as well as characteristics of the incident blast wave generated by this explosion. The accident simulation is based on the classical Bayesian approach to risk assessment. The case study described in the paper shows how to formulate the initial knowledge in line with this approach. Particular attention has been given to handling subjective information (expert opinions) within the problem under analysis. It is shown that this information is indispensable for dealing with the sparseness of hard experience data on most of the phenomena leading to an accidental explosion. The stochastic simulation demonstrated in the paper serves the purpose of propagating uncertainties related to these phenomena. The probabilistic action model describing the potential explosion takes account of these uncertainties.
\end{abstract}

Keywords: road accident, collision, explosion, aleatory uncertainty, epistemic uncertainty, simulation, nested loop.

\section{Introduction}

This paper illustrates the theoretical discussion about the simulation-based forecasting mechanical effects of accidental explosions which can occur during a road transportation of explosive goods and materials (see the fist part of the paper [1]). The present, second part of the paper describes a practical application of the procedure proposed to deal with uncertainties related to the mechanical effects of the explosions. The main objective of the second part is to demonstrate the complexity of the simulation used to predict the explosion effects.

The paper presents a case study which considers an accident on the road triggered off by a collision of two vehicles. The case study reveals the amount and character of knowledge necessary to carry out the accident simulation. This knowledge is utilised by following the theoretical concepts embodied in the classical Bayesian approach to risk analysis. It underlies the accident simulation. Results of this simulation are also expressed in line with that approach. According to it, expert opinions (subjective knowledge) make up much of input information used to the simulation. The case study demonstrates that a part of input information can be purely subjective if the knowledge in the form of hard experience data is not available for the analyst. It is shown how to introduce subjective information in the final result of the simulation, namely, a probabilistic model describing the mechanical effects of the accidental explosion.

The second part of the paper applies concepts, symbols, and abbreviations introduced in the first part [1]. Therefore, an explanation is given only to those mathematical symbols and abbreviations which are introduced in the second part.

\section{The situation under analysis}

The simulation-based procedure suggested in the first part of the paper for selecting the pam $F r_{X}(\boldsymbol{x})$ will be illustrated by considering a situation shown in Fig 1. The pam $F_{X}(\boldsymbol{x})$ is to be selected to describe peak positive overpressure $x_{1}$, positive impulse $x_{2}$, and angle in incidence $x_{3}$ of an incident blast wave which can be generated by AE. This can occur on a $150 \mathrm{~m} \times 10 \mathrm{~m}$ road segment and can be triggered off by a collision of vehicle carrying explosives (vehicle A) with another vehicle (vehicle B). 
The three characteristics of the incident blast wave are to be estimated for the point " $\Phi$ " on the facade of the building shown in Fig 1. The pam $\operatorname{Fr}_{\boldsymbol{X}}(\boldsymbol{x})$ is intended for using it for the estimation of the damage to this building.

The situation of exposure to AE shown in Fig 1 can be extended with relative ease to other geometric designs of roadways. As for the source of explosion, the case study describes a specific situation, in which effects of the distant explosion can be predicted by adapting mathematical models developed for TNT explosive. However, models are available for other types of military and commercial explosives as well as other types of blasts, say, gaseous explosions (see [2-4] and the references cited therein). The model $F_{X}(\boldsymbol{x})$ chosen in the case study for the faēade point " $\Phi$ " shown is of general character and can be applied to further assessment of potential damage, depending on the configuration and structure the exposed building.

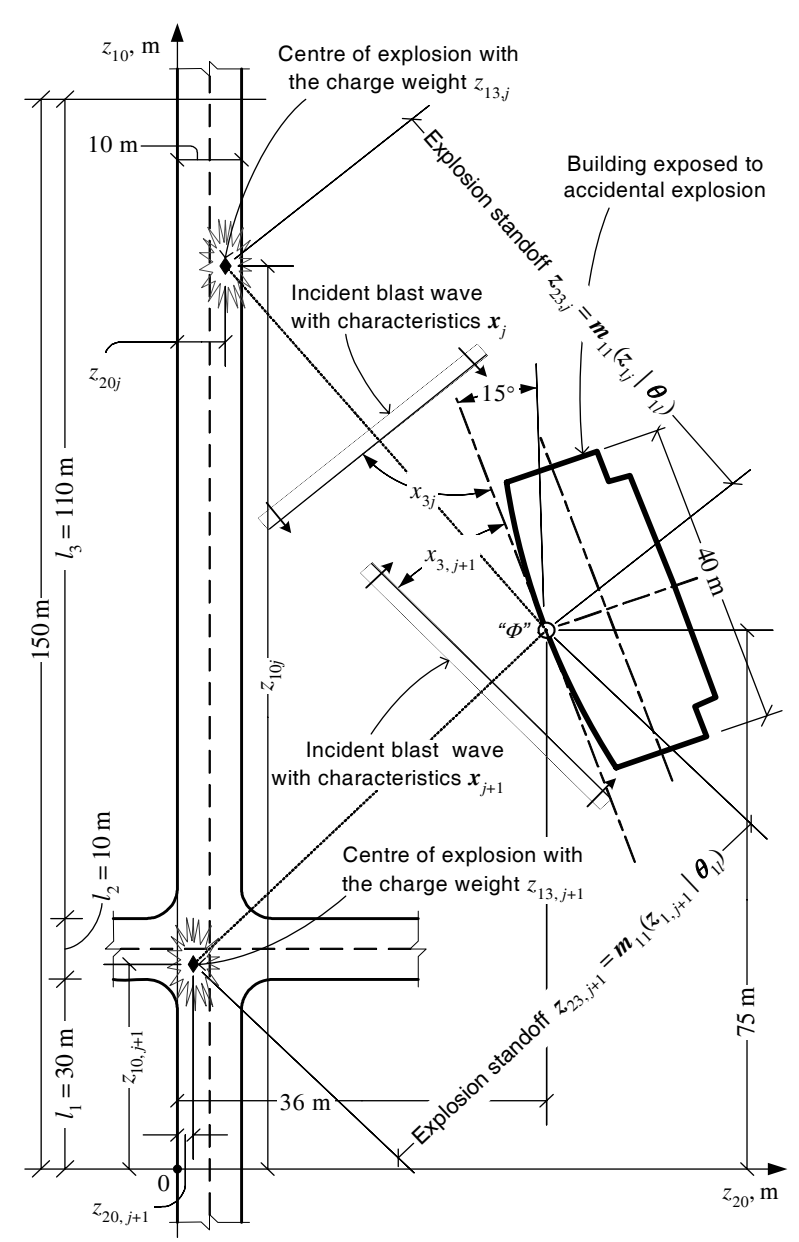

Fig 1. Situation of accident involving a collision of two vehicles and explosion in consequence of vehicular impact (the situation corresponds to the $j$ th and $(j+1)$ th repetitions of the nested loop and lth repetition of the outer loop)

\section{Modelling physical phenomena leading to an accident on the road}

\subsection{Modelling the collision of vehicles}

The model $M_{0}\left(z_{0} \mid \theta_{0}, p_{0}\right)$ describes the initiating event $E_{0}$ (the collision of vehicles, Fig 2 in [1]). It is used to express an aleatory uncertainty related to $E_{0}$ and given by the set $\left\{p_{0}, F_{0}\left(z_{0} \mid \theta_{0}\right)\right\}$. In this set, $F_{0}\left(z_{0} \mid \theta_{0}\right)$ is the joint cdf defined as a product of marginal cdf's $F_{Z_{i 0}}\left(z_{i 0} \mid \theta_{i 0}\right)$ with the parameter vectors $\theta_{i 0}(i=1,2, \ldots, 6)$, where $Z_{i 0}$ are rv's used to model the aleatory uncertainty in collision characteristics (components of $z_{0}$ ).

The arguments of $M_{0}\left(z_{0} \mid \theta_{0}, p_{0}\right)$ are $z_{0}=\left(z_{10}\right.$, $\left.z_{20}, \ldots, z_{60}\right)^{T}$, where $z_{10}$ and $z_{20}$ is the collision coordinates $(\mathrm{m})$, see Fig $1 ; z_{30}$ is the speed of vehicle $\mathrm{A}$ at a collision moment $(\mathrm{m} / \mathrm{s}) ; z_{40}$ is the speed of vehicle B at the collision moment $(\mathrm{m} / \mathrm{s}) ; z_{50}$ is the mass of vehicle $\mathrm{B}(\mathrm{kg}) ; z_{60}$ is the mass of the explosive in vehicle A (charge mass) (kg). Pd's expressing the aleatory uncertainty in components of $z_{0}$ are specified in Table 1 .

The uncertainty in the collision frequency $p_{0}$ is expressed by a rv $P_{0}$. In principle, an epistemic pd of $P_{0}$ can be selected by developing a fault tree for the "top event" $E_{0}$. This tree diagram could trace back the causes of $E_{0}$ (e.g. [5]). A quantitative analysis of the fault tree in the framework of CBA can yield an epistemic pd of $P_{0}$, that is, the cdf $F_{P_{0}}\left(p_{0} \mid \theta_{P_{0}}\right)$ [6]. However, a "full-scale" fault tree analysis in line with CBA could take a great deal of space and is beyond the scope of the present paper. Here it is simply assumed that $P_{0}$ has a gamma distribution with the parameter vector (alpha and beta) $\theta_{P_{0}}=\left(2,10\right.$ year), that is, $P_{0} \sim G(2,10)$ (the mean, mode and standard deviation of $P_{0}$ are equal to 0.2 year $^{-1}, 0.1$ year $^{-1}$, and 0.1414 year $^{-1}$, respectively). Uncertainty in components of $\theta_{60}$ is expressed by the random vector $\Theta_{60}=\left(\Theta_{1,60}, \Theta_{2,60}\right)^{T}$ with the joint cdf $F_{\Theta_{60}}\left(\theta_{60}\right)$ given by the marginal cdf's $F_{\Theta_{i, 60}}\left(\theta_{i, 60} \mid \theta_{\Theta_{i, 60}}\right)(i=1,2)$. Epistemic pd's of $\Theta_{1,60}$ and $\Theta_{2,60}$ are specified in Table 2.

Components of $z_{0}$ serve as input variables of the subsequent models $\boldsymbol{m}_{1}\left(z_{1} \mid \theta_{1}\right)$ and $\boldsymbol{m}_{3}\left(z_{3} \mid \theta_{3}\right)$. The rv's $Z_{i 0}(i=1,2, \ldots, 7)$ are assumed to be independent ones only for simplicity's sake. The pd's of $Z_{i 0}$ s were chosen hypothetically. Assigning and updating the $F_{P_{0}}\left(p_{0} \mid \theta_{P_{0}}\right)$ and $F_{\Theta_{60}}\left(\theta_{60}\right)$ is considered in Appendix.

The $j$ th simulation of accident starts with sampling the value $z_{0 j}$ from cdf $F_{0}\left(z_{0} \mid \theta_{0 l}\right)$, where $\theta_{0 l}$ is the value of $\theta_{0}$ sampled in the $l$ th repetition of the outer loop (Fig 2 in [1]). 
Table 1. Variables expressing aleatory uncertainty in characteristics of the vehicular collision (initiating event $E_{0}$ )

\begin{tabular}{|c|c|c|}
\hline Variable & Probability distribution & Distribution parameters/Comments \\
\hline$Z_{10}$ & Combined & $\begin{array}{l}U\left(0 ; l_{i}\right)^{\text {a) }} \text { in the ranges }\left[0 ; l_{i}\right] \quad(i=1,2,3 ; \text { Fig } 1) \text { with the discrete probabilities } \\
\left\{P\left(0<Z_{10} \leq 30\right)=0,15 ; P\left(30<Z_{10} \leq 40\right)=0,4 ; P\left(40<Z_{10} \leq 150\right)=0,45\right\}\end{array}$ \\
\hline$Z_{20}$ & $U(0 ; 30)$ & $\theta_{20}=(0 \mathrm{~m} ; 10 \mathrm{~m})^{T}$ \\
\hline$Z_{30}$ & $N(13,9 ; 17,4)^{\mathrm{b})}$ & $\theta_{30}=\left(13,9 \mathrm{~m} / \mathrm{s} ; 17,4(\mathrm{~m} / \mathrm{s})^{2}\right)^{T}$ \\
\hline$Z_{40}$ & $N( \pm 22,2 ; 30,9)$ & $\begin{array}{l}\theta_{40}=\left( \pm 22,2 \mathrm{~m} / \mathrm{s} ; 30,9(\mathrm{~m} / \mathrm{s})^{2}\right)^{T} \text { (" }+" \text { " and "-" stand for velocity at frontal and rear-end colli- } \\
\text { sion, respectively) }\end{array}$ \\
\hline$Z_{50}$ & $\left.L(7,8044 ; 0,19804)^{\mathrm{c}}\right)$ & $\theta_{50}=(7,8044 ; 0,19804)^{T}($ mean and standard deviation are $2500 \mathrm{~kg}$ of $500 \mathrm{~kg})$ \\
\hline$Z_{60}$ & Discrete & $\begin{array}{l}\theta_{60} \text { is not used in the explicit form; } Z_{60} \text { is a discrete rv distributed over } 15 \mathrm{~kg}, 25 \mathrm{~kg} \text { and } \\
40 \mathrm{~kg} \text { with the probabilities }\left\{P\left(Z_{60}=15=0,3, P\left(Z_{60}=25\right)=0,3, P\left(Z_{60}=40\right)=0,4\right\}\right.\end{array}$ \\
\hline
\end{tabular}

Table 2. Probability distributions quantifying epistemic uncertainty in the parameters of the models used to the accident simulation

\begin{tabular}{|c|c|c|}
\hline Parameter & Probability distribution & Distribution parameters/Comments \\
\hline \multicolumn{3}{|c|}{ Distributions of parameters related to the model $M_{0}\left(z_{0} \mid \theta_{0}, p_{0}\right)$} \\
\hline$\Theta_{1,60}$ & $B e(25 ; 50)^{\mathrm{a})}$ & $\theta_{\theta_{1,60}}=(25 ; 50)^{T}$ (beta distribution with alpha $=25$ and beta $=50 ; Z_{60}$ is dimensionless $)$ \\
\hline$\Theta_{2,60}$ & $\operatorname{Ex}(140)^{\mathrm{b})}$ & $\theta_{\theta_{2,60}}=(140)$ (exponential distribution with the mean of 0,$00714 ; Z_{60}$ is dimensionless) \\
\hline \multicolumn{3}{|r|}{ Distributions of parameters related to the model $m_{1}\left(z_{1} \mid \theta_{1}\right)$} \\
\hline$\Theta_{11}$ & $N(6 ; 1)$ & $\theta_{\theta_{11}}=\left(6 \mathrm{~kg} \mathrm{~m}^{2} / \mathrm{s}^{2} ; 1\left(\mathrm{~kg} \mathrm{~m}^{2} / \mathrm{s}^{2}\right)^{2}\right)^{T}$ \\
\hline$\Theta_{21}^{-1}$ & $G(20 ; 19)^{\mathrm{c})}$ & $\begin{array}{l}\theta_{\Theta_{21}}=\left(20 ; 19\left(\mathrm{~kg} \mathrm{~m}^{2} / \mathrm{s}^{2}\right)^{2}\right)^{T}\left(\text { gamma distribution with the mean of } 1,05\left(\mathrm{~kg} \mathrm{~m}^{2} / \mathrm{s}^{2}\right)^{-2} \text {, mode }\right. \\
\left.\text { of } 1,05\left(\mathrm{~kg} \mathrm{~m}^{2} / \mathrm{s}^{2}\right)^{-2} \text {, and standard deviation of } 0,235\left(\mathrm{~kg} \mathrm{~m}^{2} / \mathrm{s}^{2}\right)^{-2}\right)\end{array}$ \\
\hline$\Theta_{31}$ & $B e(10 ; 20)$ & $\theta_{\Theta_{31}}=(10 ; 20)^{T}$ (beta distribution with alpha $=10$ and beta $\left.=20\right)$ \\
\hline \multicolumn{3}{|r|}{ Distributions of parameters related to the model $m_{2}\left(z_{2} \mid \theta_{2}\right)$} \\
\hline$\Theta_{12}$ & $N\left(700 \times 10^{3} ; 4,9 \times 10^{9}\right)$ & $\theta_{\theta_{12}}=\left(700 \times 10^{3} \mathrm{~kg} \mathrm{~m}^{2} / \mathrm{s}^{2} ; 4,9 \times 10^{9}\left(\mathrm{~kg} \mathrm{~m}^{2} / \mathrm{s}^{2}\right)^{2}\right)^{T}$ \\
\hline$\Theta_{22}$ & $N\left(7 \times 10^{4} ; 12,25 \times 10^{6}\right)$ & $\theta_{\theta_{22}}=\left(7 \times 10^{4} \mathrm{~kg} \mathrm{~m}^{2} / \mathrm{s}^{2} ; 12,25 \times 10^{6}\left(\mathrm{~kg} \mathrm{~m}^{2} / \mathrm{s}^{2}\right)^{2}\right)^{T}$ \\
\hline \multicolumn{3}{|r|}{ Distributions of parameters of the model $\boldsymbol{m}_{3}\left(z_{3} \mid \theta_{3}\right)$} \\
\hline$\Theta_{13}$ & $N\left(-0,125 ; 1,56 \times 10^{-4}\right)$ & $\theta_{\theta_{13}}=\left(-0,125 ; 1,56 \times 10^{-4}\right)^{T}$ (the parent variable $\Pi^{\prime \prime}$ is dimensionless quantity) \\
\hline$\Theta_{23}$ & $G(15 ; 0,6)$ & $\theta_{\theta_{23}}=(15 ; 0,6)^{T}$ (the parent variable $\Pi^{\prime \prime}$ is dimensionless quantity) \\
\hline$\Theta_{33}$ & $N\left(-0,159 ; 2,53 \times 10^{-4}\right)$ & $\theta_{\theta_{33}}=\left(-0,159 ; 2,53 \times 10^{-4}\right)^{T}$ (the parent variable $\Pi^{\prime \prime \prime}$ is dimensionless quantity) \\
\hline$\Theta_{43}$ & $N(15 ; 0,6)$ & $\theta_{\theta_{43}}=(15 ; 0,6)^{T}$ (the parent variable $\Pi^{\prime \prime \prime}$ is dimensionless quantity) \\
\hline$\Theta_{53}$ & $N\left(0,1 ; 1,0 \times 10^{-4}\right)$ & $\theta_{\Theta_{53}}=\left(0,1 \mathrm{MPa} \mathrm{m} / \mathrm{kg}^{1 / 3} ; 1,0 \times 10^{-4}\left(\mathrm{MPa} \mathrm{m} / \mathrm{kg}^{1 / 3}\right)^{2}\right)^{T}$ \\
\hline$\Theta_{63}$ & $N\left(0,43 ; 1,6 \times 10^{-3}\right)$ & $\theta_{\theta_{63}}=\left(0,43 \mathrm{MPa} \mathrm{m} / \mathrm{kg}^{2 / 3} ; 1,6 \times 10^{-3}\left(\mathrm{MPa} \mathrm{m}^{2} / \mathrm{kg}^{2 / 3}\right)^{2}\right)^{T}$ \\
\hline$\Theta_{73}$ & $N\left(1,4 ; 2,25 \times 10^{-2}\right)$ & $\theta_{\theta_{73}}=\left(1,4 \mathrm{MPa} \mathrm{m}^{3} / \mathrm{kg} ; 2,25 \times 10^{-2}\left(\mathrm{MPa} \mathrm{m}^{3} / \mathrm{kg}\right)^{2}\right)^{T}$ \\
\hline$\Theta_{83}$ & $N(6,3 ; 0,36)$ & $\theta_{\theta_{83}}=\left(6,3 \mathrm{MPa} \mathrm{s} /\left(\mathrm{m} \mathrm{kg}^{2 / 3}\right) ; 0,36\left(\mathrm{MPa} \mathrm{s} /\left(\mathrm{m} \mathrm{kg}^{2 / 3}\right)\right)^{2}\right)^{T}$ \\
\hline
\end{tabular}

\subsection{Modelling the exceedance of the tolerable value of collision energy}

The model $\boldsymbol{m}_{1}\left(z_{1} \mid \theta_{1}\right)$ is related to a possible exceedance of the tolerable value of collision energy which can lead to an explosion in vehicle A. The exceedance is represented by the re $E_{1}$ (Figs 1 and 2 in [1]). $\boldsymbol{m}_{1}\left(z_{1} \mid \theta_{1}\right)$ is used to decide whether the collision energy is sufficient to damage a container with the explosive charge and trigger off the explosion as well as to compute standoff of the explosion (Fig 1).

Model input is expressed as $z_{1}=\left(z_{11}, z_{21}, \ldots, z_{61}\right)^{T}$, where $z_{11}$ and $z_{21}$ are the collision coordinates $(\mathrm{m}) ; z_{31}$ and $z_{41}$ are the velocities of vehicles $\mathrm{A}$ and $\mathrm{B}$ at the collision moment $(\mathrm{m} / \mathrm{s}) ; z_{51}$ and $z_{61}$ are the masses of vehicles $\mathrm{A}$ and $\mathrm{B}(\mathrm{kg})$. Values of arguments of the preceding model $M_{0}\left(z_{0} \mid \theta_{0}, p_{0}\right)$ are assigned to components of $z_{1}$ as follows: $z_{i 1}=z_{i 0}(i=1,2,3,4)$; $z_{51}=6000 \mathrm{~kg}+z_{60} ; z_{61}=z_{50}$. Here the value $6000 \mathrm{~kg}$ is the mass of vehicle A without the load of explosive.

Model output is given by the vector $\boldsymbol{m}_{1}=\left(m_{11}\right.$, $\left.m_{21}, m_{31}\right)^{T}$, where $m_{11}$ is the standoff $(\mathrm{m}) ; m_{21}$ is the collision energy $\left(\mathrm{kg} \mathrm{m}^{2} / \mathrm{s}^{2}\right) ; m_{31}$ is the difference between the collision energy and a "threshold" energy value which can be tolerated by vehicle $\mathrm{A}$ without leading to an explosion $\left(\mathrm{kg} \mathrm{m}^{2} / \mathrm{s}^{2}\right)$.

The structure of $\boldsymbol{m}_{1}\left(z_{1} \mid \theta_{1}\right)$ is as follows: 


$$
\left\{\begin{array}{l}
m_{11}\left(z_{1}\right)=\left(\left(75-z_{11}\right)^{2}+\left(36-z_{21}\right)^{2}\right)^{1 / 2} \\
m_{21}\left(z_{1} \mid \theta_{1}\right)=\frac{z_{51} z_{61}}{2\left(z_{51}+z_{61}\right)} \frac{\left(1-\theta_{31}\right)}{\left(z_{31}+z_{41}\right)^{-2}} \\
m_{31}\left(z_{1} \mid \theta_{1}\right)=m_{21}\left(z_{1}\right)-\theta^{\prime} ; F_{\theta^{\prime}}\left(\theta^{\prime} \mid \theta_{11}, \theta_{21}\right)
\end{array}\right\},
$$

where $\theta^{\prime}$ is the "threshold" energy value $\left(\mathrm{kg} \mathrm{m}^{2} / \mathrm{s}^{2}\right)$; $F_{\Theta^{\prime}}\left(\theta^{\prime} \mid \theta_{11}, \theta_{21}\right)$ is the cdf of rv $\Theta^{\prime}$ used to model an aleatory uncertainty in $\theta^{\prime} ; \theta_{31}$ is the dimensionless parameter expressing a mechanical behaviour of vehicles $\mathrm{A}$ and $\mathrm{B}$ at the collision $\left(0 \leq \theta_{31} \leq 1\right.$; if $\theta_{31}=0$, the vehicles are considered perfectly plastic bodies; if $\theta_{31}=1$, the vehicles are considered perfectly elastic bodies). It is assumed that $\left(\Theta^{\prime} \times 10^{-5}\right) \sim N\left(\theta_{11}, \theta_{21}\right)$, where $\theta_{11}$ and $\theta_{21}$ are the mean and variance of a normal pd, respectively. Components of $\theta_{1}=$ $\left(\theta_{11}, \theta_{21}\right)^{T}$ are considered to be uncertain in the epistemic sense. Uncertainty in $\theta_{1}$ is expressed by the random vector $\Theta_{1}=\left(\Theta_{11}, \Theta_{21}, \Theta_{31}\right)^{T}$ with the joint c.d.f $F_{\theta_{1}}\left(\theta_{1}\right)$ given by the marginal cdf's $F_{\theta_{i 1}}\left(\theta_{i 1} \mid \theta_{\theta_{i 1}}\right)$ $(i=1,2,3)$. Epistemic pd's of $\Theta_{11}$ to $\Theta_{31}$ are specified in Table 2.

The model component $m_{31}\left(z_{1} \mid \theta_{1}\right)$ is used to decide which of the events $E_{1}$ or $\bar{E}_{1}$ will occur:

$$
\mathbf{1}\left(z_{1 j} \mid \boldsymbol{\theta}_{1 l}\right)= \begin{cases}1 & \text { if } m_{31}\left(z_{1 j} \mid \boldsymbol{\theta}_{1 l}\right) \geq 0\left(E_{1} \text { occurs }\right) \\ 0 & \text { if } m_{31}\left(z_{1 j} \mid \boldsymbol{\theta}_{1 l}\right)<0\left(\bar{E}_{1} \text { occurs }\right)\end{cases}
$$

where $z_{1 j}$ and $\theta_{j}^{\prime}$ are the values of $z_{1}$ and $\theta^{\prime}$, respectively, used in the $j$ th repetition of the nested loop; $\theta_{1 l}$ is a value of $\theta_{1}$ sampled from the cdf $F_{\theta^{\prime}}\left(\theta^{\prime} \mid \theta_{1}\right)$ in the $l$ th repetition of the outer loop. The value $z_{1 j}$ is obtained by sampling from the model $M_{0}\left(z_{0} \mid \theta_{0}, p_{0}\right)$.

The model $\boldsymbol{m}_{1}\left(z_{1} \mid \theta_{1}\right)$ is underpinned by the following assumptions made for the sake of simplicity: (i) Frontal and rear-end collision of vehicles A and B is possible in the segments $l_{1}$ and $l_{3}$ (Fig 1 ); the conditional probabilities of the frontal and rear-end impact given a collision are 0,4 and 0,6 , respectively;

(ii) Collision of vehicles A and B at right angle is possible in the segment $l_{2}$ in addition to the frontal and rear-end collision; the conditional probabilities of all three types of impact given a collision are $0,5,0,2$, and 0,3 , respectively; the energy of the side impact by the vehicle $\mathrm{B}$ is determined by mass and velocity of this vehicle.

The conditional probabilities assumed above should be considered elements of one of the models $M_{0}\left(z_{0} \mid \theta_{0}, p_{0}\right)$ or $\boldsymbol{m}_{1}\left(z_{1} \mid \theta_{1}\right)$. In the context of CBA these probabilities should be treated as measures of aleatory uncertainty. In principle, measures of epistemic uncertainty can be assigned to them; however, this is not done in the present case study for brevity.
The expression of the collision energy, $m_{21}\left(z_{1}\right)$, was adopted from [7, 8]. The normal pd of $\Theta^{\prime}$ was chosen hypothetically. Assigning and updating the pd represented by cdf $F_{\theta_{1}}\left(\theta_{1}\right)$ is considered in Appendix.

\subsection{Modelling the explosion of the charge in vehicle}

The explosion in vehicle $\mathrm{A}$ is represented by the re $E_{2}$ (see Figs 1 and 2 in [1]). The model $m_{2}\left(z_{2} \mid \theta_{2}\right)$ serves for a simulation of the occurrence or nonoccurrence of $E_{2}$. In particular, $m_{2}\left(z_{2} \mid \theta_{2}\right)$ is used to decide whether the collision energy, when it exceeds the safe "threshold" value, will cause the explosion.

Model input is represented by the collision energy $z_{2}\left(\mathrm{~kg} \mathrm{~m}^{2} / \mathrm{s}^{2}\right)$. Values of $z_{2}$ are assigned by $z_{2}=m_{21}\left(z_{1}\right)$. Model output is an auxiliary dimensionless variable $m_{2}$ used in the decision rule related to $m_{2}\left(z_{2} \mid \theta_{2}\right)$. The structure $m_{2}\left(z_{2} \mid \theta_{2}\right)$ is:

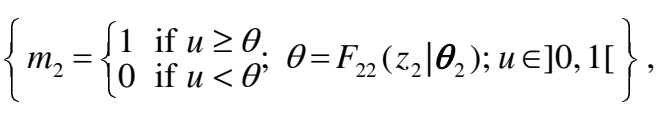

where $u$ is an auxiliary variable uniformly distributed over the interval $] 0 ; 1\left[; F_{22}\left(z_{2} \mid \theta_{2}\right)\right.$ is the fragility function (cdf of a normal pd with the parameter vector $\theta_{2}$ ); the parameter $\theta$ models an explosion probability. The output variable $m_{2}$ models an occurrence or non-occurrence of the explosion; $m_{2}$ can take on values 1 (event $E_{2}$ occurs) or 0 (event $\bar{E}_{2}$ occurs).

Components of $\theta_{2}$ are considered to be uncertain in the epistemic sense. Uncertainty in $\theta_{2}$ is expressed by the random vector $\Theta_{2}=\left(\Theta_{12}, \Theta_{22}\right)^{T}$ with the joint cdf $F_{\Theta_{2}}\left(\theta_{2}\right)$ expressed as a product of the marginal cdf's $F_{\theta_{i 2}}\left(\theta_{i 2} \mid \theta_{\theta_{i 2}}\right) \quad(i=1,2)$. Epistemic pd's of $\Theta_{12}$ and $\Theta_{22}$ are specified in Table 2 .

The value $m_{2}\left(z_{2 j} \mid \theta_{2 l}\right)$ is used to decide which of the events $E_{2}$ or $\bar{E}_{2}$ will take place:

$$
\mathbf{1}\left(z_{2 j} \mid \boldsymbol{\theta}_{2 l}\right)=\left\{\begin{array}{lll}
1 & \text { if } \left.u_{j} \geq F_{22}\left(z_{2 j} \mid \theta_{2 l}\right)\right) & \left(E_{2} \text { occurs }\right), \\
0 & \text { if } \left.u_{j}<F_{22}\left(z_{2 j} \mid \theta_{2 l}\right)\right) & \left(\bar{E}_{2} \text { occurs }\right),
\end{array}\right.
$$

where $z_{2 j}$ is the $j$ th collision energy value; $u_{j}$ is the $j$ th value of $u$ sampled from the uniform pd $U(0,1)$; and $\theta_{2 l}$ is the value of $\theta_{2}$ sampled in the $l$ th repetition of the outer loop.

The model $m_{2}\left(z_{2} \mid \pi_{2}\right)$ is underpinned by the assumption that an exceedance of the "threshold" energy value $\theta^{\prime}$ used in the model $\boldsymbol{m}_{1}\left(z_{1} \mid \theta_{1}\right)$ does not necessarily cause an explosion of the charge in vehicle A. However, the probability of such an explosion, $\theta$, is the higher the larger is the collision energy $z_{2}$. The cdf of a normal pd was chosen as the fragility function $F_{22}\left(z_{2} \mid \theta_{2}\right)$ hypothetically. Assigning and updating the cdf $F_{\theta_{2}}\left(\theta_{2}\right)$ is considered in Appendix. 


\subsection{Modelling used to predict characteristics of the incident blast wave}

The model $\boldsymbol{m}_{3}\left(z_{3} \mid \theta_{3}\right)$ serves for predicting characteristics of the incident blast wave represented by the vector $\boldsymbol{x}$ (Fig 1). This model is used to relate the charge mass and position of explosion centre to $\boldsymbol{x}$.

Input $\boldsymbol{m}_{3}\left(z_{3} \mid \theta_{3}\right)$, is represented by $z_{3}=\left(z_{13}\right.$, $\left.z_{23}, z_{33}, z_{43}\right)^{T}$, where $z_{13}$ and $z_{23}$ are the charge mass $(\mathrm{kg})$ and standoff $(\mathrm{m})$, respectively; $z_{33}$ and $z_{43}$ are the coordinates of explosion centre. Values are assigned to components of $z_{3}$ by the expressions $z_{13}=z_{60}, \quad z_{23}=m_{11}\left(z_{1}\right), \quad z_{33}=z_{10}$, and $z_{43}=z_{20}$. Model output is given by the vector $\boldsymbol{m}_{3}=\left(m_{13}, m_{23}\right.$, $\left.m_{33}\right)^{T}$, the components of which are peak positive overpressure (MPa) positive impulse $\left(\mathrm{MPa} \mathrm{s} / \mathrm{m}^{2}\right)$, and angle of incidence (degrees), respectively. In terms of the notation used in Sec. 4 of the first part of this paper, $x_{1}=m_{13}, x_{2}=m_{23}$ and $x_{3}=m_{33}$ [1].

The model $\boldsymbol{m}_{3}\left(z_{3} \mid \theta_{3}\right)$ has the following structure:

$$
\left\{\begin{array}{l}
m_{13}\left(z_{3} \mid \theta_{3}\right)=\left\{\begin{array}{l}
m_{13}=\pi^{\prime \prime} m_{13}^{\prime}\left(z_{3} \mid \theta_{53}, \theta_{63}, \theta_{73}\right) \\
F_{\ln \Pi^{\prime \prime}}\left(\ln \pi^{\prime \prime} \mid \theta_{13}, \theta_{23}\right)
\end{array}\right\} \\
m_{23}\left(z_{3} \mid \theta_{3}\right)=\left\{\begin{array}{l}
m_{13}=\pi^{\prime \prime} m_{13}^{\prime}\left(z_{3} \mid \theta_{53}, \theta_{63}, \theta_{73}\right) \\
F_{\ln \Pi^{\prime \prime}}\left(\ln \pi^{\prime \prime} \mid \theta_{13}, \theta_{23}\right)
\end{array}\right\} \\
m_{33}\left(z_{3}\right)=\left|\tan ^{-1}\left\{\frac{\left|75-z_{33}\right|}{36-z_{43}}\right\}-\operatorname{sign}\left\{75-z_{33}\right\} 15^{\circ}\right|
\end{array}\right\}
$$

with

$$
\begin{aligned}
& m_{13}^{\prime}\left(\mathrm{z}_{3} \mid \theta_{53}, \theta_{63}, \theta_{73}\right)=\theta_{53} \frac{z_{13}^{1 / 3}}{z_{23}}+\theta_{63} \frac{z_{13}^{2 / 3}}{z_{23}^{2}}+\theta_{73} \frac{z_{13}}{z_{23}^{3}}, \\
& m_{23}^{\prime}\left(\mathrm{z}_{3} \mid \theta_{83}\right)=\theta_{83} \frac{z_{13}^{2 / 3}}{z_{23}},
\end{aligned}
$$

where $\pi^{\prime \prime}$ and $\pi^{\prime \prime \prime}$ are the dimensionless adjustment factors (relative overpressure and relative impulse of the explosive in vehicle A compared to an equivalent weight of TNT explosive); $m_{13}^{\prime}\left(z_{3} \mid \theta_{53}, \theta_{63}, \theta_{73}\right)$ and $m_{23}^{\prime}\left(z_{3} \mid \theta_{83}\right)$ are the models relating components of $z_{3}$ to the overpressure and impulse of TNT explosion, respectively; $\quad F_{\ln \Pi^{\prime \prime}}\left(\ln \pi^{\prime \prime} \mid \theta_{13}, \theta_{23}\right) \quad$ and $F_{\ln \Pi^{\prime \prime \prime}}\left(\ln \pi^{\prime \prime \prime} \mid \pi_{33}, \pi_{43}\right)$ are the cdf's expressing an aleatory uncertainty in logarithms of the factors $\pi^{\prime \prime}$ and $\pi^{\prime \prime \prime}$. The rv's $\Pi^{\prime \prime}$ and $\Pi^{\prime \prime \prime}$ are used to model the aleatory uncertainty: $\ln \Pi^{\prime \prime} \sim N\left(\theta_{13}, \theta_{23}\right)$ (a normal distribution with an uncertain mean $\theta_{13}$ and variance $\left.\theta_{23}\right) ; \ln \Pi^{\prime \prime \prime} \sim N\left(\theta_{33}, \theta_{43}\right)$ (a normal distribution with an uncertain mean $\theta_{33}$ and variance $\left.\theta_{43}\right)$.

Components of $\theta_{3}$ (distribution parameters $\theta_{13}, \theta_{23}, \theta_{33}, \theta_{43}$ and regression parameters $\theta_{53}, \theta_{63}$, $\left.\theta_{73}, \theta_{83}\right)$ are considered to be uncertain in the epistemic sense. Uncertainty in $\theta_{3}$ is expressed by the random vector $\Theta_{3}=\left(\Theta_{13}, \Theta_{23}, \ldots, \Theta_{83}\right)^{T}$ with the joint cdf $F_{\Theta_{3}}\left(\theta_{3}\right)$ defined through the marginal cdf's $F_{\theta_{i 3}}\left(\theta_{i 3} \mid \theta_{\Theta_{i 3}}\right)(i=1,2, \ldots, 8)$. Pd's of components of $\Theta_{3}$ are specified in Table 2 .

The models $m_{13}^{\prime}\left(z_{3} \mid \theta_{53}, \theta_{63}, \theta_{73}\right)$ and $m_{23}^{\prime}\left(z_{3} \mid \theta_{83}\right)$ were adopted from [9]. The lognormal pd's of the random adjustment factors $\Pi^{\prime \prime}$ and $\Pi^{\prime \prime \prime}$ were chosen hypothetically. Deterministic values of these factors suitable to an adjustment of the TNT models $m_{13}^{\prime}\left(z_{3} \mid \theta_{53}, \theta_{63}, \theta_{73}\right)$ and $m_{23}^{\prime}\left(z_{3} \mid \theta_{83}\right)$ can be found, e.g., in [10]. Assigning and updating the cdf $F_{\theta_{3}}\left(\theta_{3}\right)$ is considered in Appendix.

\section{The process and results of accident simulation}

The accident on the road was simulated with $n_{p}=300$ and $n_{0}=1000$. The simulation generated a sample of frequencies, $p_{0 l} n_{a l} / n_{0}(l=1,2, \ldots, 300)$, and samples of action characteristics, $\mathbf{x}_{l} \quad(l=1,2$, $\ldots, 300)$. Descriptive measures of the samples consisting of the values $n_{a l}$ and $p_{0 l} n_{a l} / n_{0}$ are given in Table 3. A gamma distribution with the parameter vector $\theta_{a}=(1,341 ; 19,69$ year $)$ can be fitted to the sample $\quad p_{0 l} n_{a l} / n_{0} \quad(l=1,2, \ldots, 300)$ as cdf $F_{P_{a}}\left(p_{a} \mid \theta_{P_{a}}\right)$ (Fig $\left.2 \mathrm{a}\right)$. This distribution expresses the epistemic uncertainty in the explosion frequency $p_{a}$.

Table 3. Descriptive measures of samples related to the likelihood of occurrence of accidental explosion on the road

\begin{tabular}{lcc}
\hline $\begin{array}{c}\text { Descriptive } \\
\text { measure }\end{array}$ & $\begin{array}{c}\text { Sample } n_{a l} \\
(l=1, \ldots, 300)\end{array}$ & $\begin{array}{c}\text { Sample } p_{0 l} n_{a l} / n_{0}(l=1, \\
\left.\ldots, 300 ; n_{0}=1000\right)\end{array}$ \\
\hline $\begin{array}{l}\text { Mean } \\
\text { Coefficient }\end{array}$ & 338 & 0.0682 year $^{-1}$ \\
of variables & $0,486 \%$ & $0,873 \%$ \\
Minimum & 4 & $0,465 \times 10^{-3}$ year $^{-1}$ \\
Maximum & 731 & 0,3778 year $^{-1}$ \\
Skewness & 0,147 & 1,83 \\
Kurtosis & $-0,729$ & 4,72 \\
\hline
\end{tabular}

The cdf's $F_{\boldsymbol{X}_{i}}\left(\boldsymbol{x} \mid \boldsymbol{\theta}_{x i}\right) \quad(i=1,2,3)$ can be chosen by applying the heuristic procedure suggested in $[11,12]$. This procedure requires to preset number $n$ and probabilistic weights $p_{i}(i=1,2, \ldots, n)$ in advance. In the present case study, it is assumed that $n=3$ and $p_{1}=p_{3}=0,3, p_{2}=0,4$. With these values, the samples $\mathbf{x}_{l}(l=1,2, \ldots, 300)$ were grouped in three clusters $\mathbf{x}_{i}^{\prime}(i=1,2,3)$ with descriptive measures given in Tables 4 and 5 . Fig 2 b, c, d shows three 
Table 4. Descriptive measures of the clusters $\mathbf{x}_{i}^{\prime}$ obtained by grouping the simulated samples $\mathbf{x}_{l}(i=1,2,3)$

\begin{tabular}{ccccccccc}
\hline $\begin{array}{c}\text { No. of } \\
\text { cluster } i\end{array}$ & $\begin{array}{c}\text { Weight } \\
\mathrm{p}_{\mathrm{i}}\end{array}$ & $\begin{array}{c}\text { Size of } \\
\text { cluster } n_{c i}\end{array}$ & $\begin{array}{c}\text { Mean of } \\
\text { cluster }\end{array}$ & $\begin{array}{c}\text { Coef. of } \\
\text { var. }(\%)\end{array}$ & Skewness & Kurtosis & Min & Max \\
\hline \multicolumn{8}{c}{ Simulated values of the initial overpressure $X_{1}(\mathrm{MPa})$} \\
\hline 1 & 0,3 & 37258 & $8,90 \times 10^{-3}$ & 47,2 & 1,35 & 2,40 & $1,85 \times 10^{-3}$ & $35,1 \times 10^{-3}$ \\
2 & 0,4 & 36444 & $7,58 \times 10^{-3}$ & 44,5 & 1,26 & 1,72 & $1,78 \times 10^{-3}$ & $25,0 \times 10^{-3}$ \\
3 & 0,3 & 27723 & $6,82 \times 10^{-3}$ & 47,4 & 1,42 & 2,75 & $1,70 \times 10^{-3}$ & $27,6 \times 10^{-3}$ \\
\hline \multicolumn{8}{c}{ Simulated values of the initial impulse $X_{2}(\mathrm{MPa}$ s/m2) } \\
\hline 1 & 0,3 & 37258 & 1,231 & 46,1 & 1,11 & 1,544 & 0,264 & 4,499 \\
2 & 0,4 & 36444 & 1,007 & 41,2 & 0,927 & 0,754 & 0,263 & 2,929 \\
3 & 0,3 & 27723 & 0,908 & 42,6 & 1,03 & 1,201 & 0,238 & 2,927 \\
\hline \multicolumn{8}{c}{ Simulated values of the angle of incidence $X_{3}$ (degrees) } \\
\hline 1 & 0,3 & 37258 & 43,94 & 44,9 & 0,156 & $-0,360$ & 0,00409 & 85,8 \\
3 & 0,4 & 36444 & 43,82 & 45,2 & 0,160 & $-0,374$ & 0,00013 & 85,7 \\
\end{tabular}

Table 5. Correlation matrices of the components constituting the clusters $\mathbf{x}_{i}^{\prime}(i=1,2,3)$

\begin{tabular}{clll}
\hline No. of cluster $i$ & 1 & \multicolumn{1}{c}{2} & 3 \\
\hline Correlation matrix & {$\left[\begin{array}{llll}1 & 0,609 & -0,533 \\
0,609 & 1 & -0,421 \\
-0,533 & -0,421 & 1\end{array}\right] \quad\left[\begin{array}{llll}1 & 0,846 & -0,568 \\
0,846 & 1 & -0,468 \\
-0,568 & -0,468 & 1\end{array}\right] \quad\left[\begin{array}{llll}1 & 0,725 & -0,529 \\
0,725 & 1 & -0,438 \\
-0,529 & -0,438 & 1\end{array}\right]$} \\
\hline
\end{tabular}

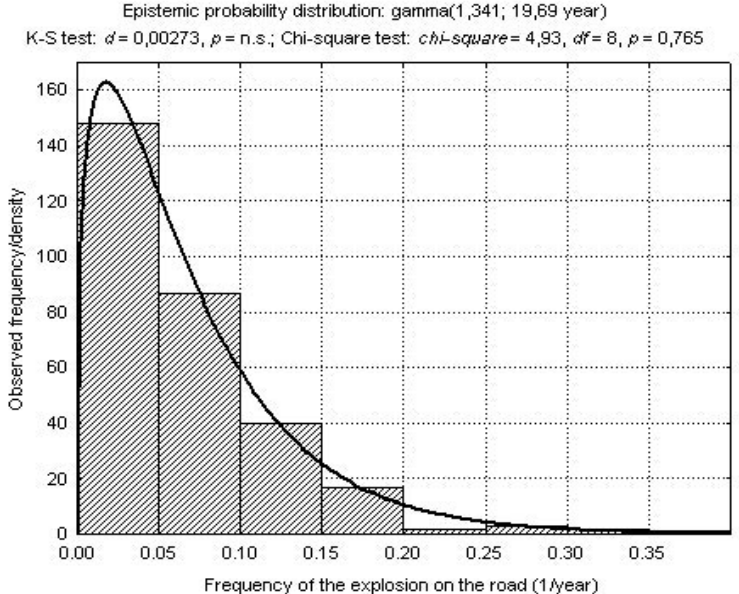

(a)

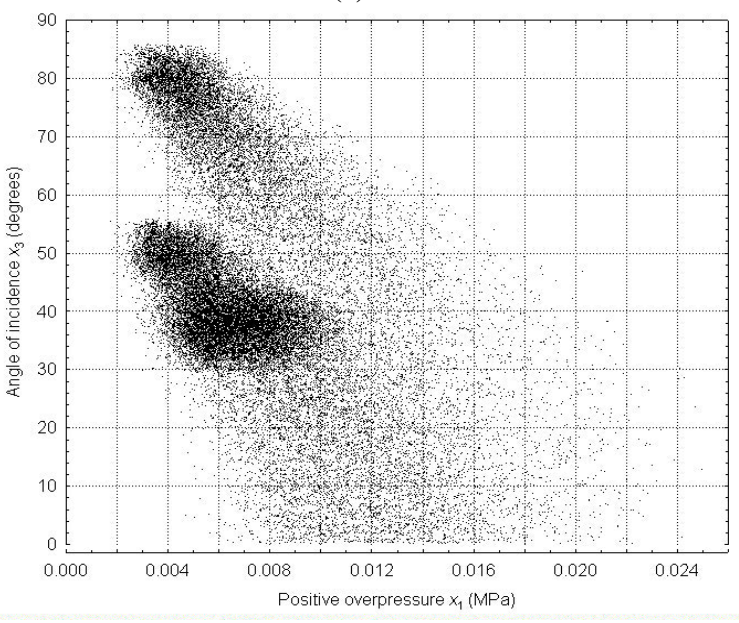

(c)

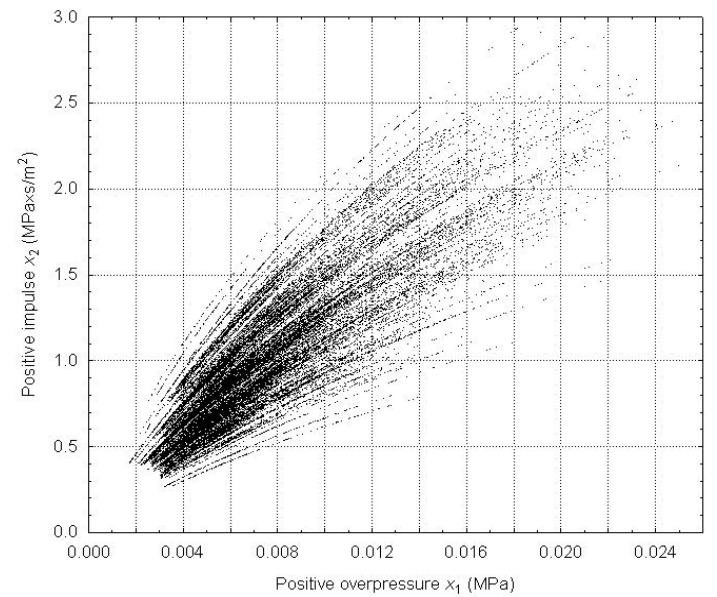

(b)

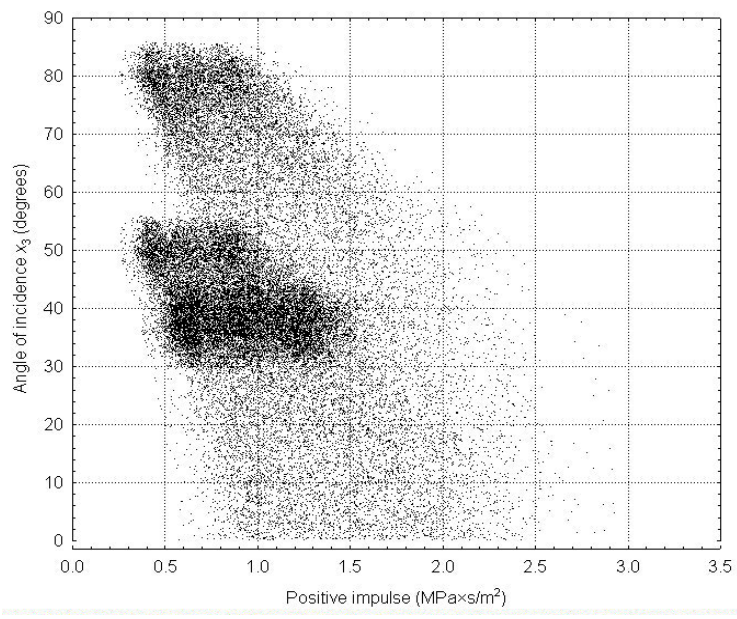

(d)

Fig 2. Diagrams showing results obtained by a simulation of accident on the road: (a) fitting a gamma distribution used to express epistemic uncertainty in the frequency of explosion; (b)...(d) scatter diagrams of the simulated characteristics of the incident blast wave, $x_{1}, x_{2}$, and $x_{3}$, drawn for the second cluster $\mathbf{x}_{2}^{\prime}$ 
scatter diagrams of the second cluster $\mathbf{x}_{2}^{\prime}$. The size of the second cluster, $n_{c 2}$, (number of points in the diagrams (b) to (d) given in Fig 2) is equal to 36444 (see Table 4). Scatter diagrams of $\mathbf{x}_{1}^{\prime}$ and $\mathbf{x}_{3}^{\prime}$ are similar to the ones shown in Fig 2.

From Table 5 and Fig 2 it may be deduced that there exists relatively complicated dependence between the blast wave characteristics $X_{i}(i=1,2,3)$. Thus fitting a widely known three-dimensional pd to the clusters $\mathbf{x}_{i}^{\prime}(i=1,2,3)$ is problematic. In theory, one can apply complicated transformations of $\mathbf{x}_{i}^{\prime}$ in order to make fitting a standard distribution possible [11]. However, it is not always possible to set up such transformations.

A simple, practical alternative to the formal fitting cdf's $F_{X_{i}}\left(\boldsymbol{x} \mid \theta_{x i}\right)$ to the clusters $\mathbf{x}_{i}^{\prime}$ is describing them by empirical distribution functions $\hat{F}_{i}(\boldsymbol{x})$. They can represent corresponding clusters $\mathbf{x}_{i}^{\prime}$ with reasonable accuracy and so can replace cdf's $F_{X_{i}}\left(x \mid \theta_{x i}\right)$ in the action model defined by Equation given in the first part of the paper [1]. The result of such a substitution will be the model:

$$
\begin{gathered}
F r_{X}(\boldsymbol{x})=\left\{F_{P_{a}}\left(p_{a} \mid \theta_{\alpha}\right),\left(\hat{F}_{1}(\boldsymbol{x}), 0,3\right),\right. \\
\left.\left(\hat{F}_{2}(\boldsymbol{x}), 0,4\right),\left(\hat{F}_{3}(\boldsymbol{x}), 0,3\right)\right\} .
\end{gathered}
$$

The pam $F_{X_{X}}(\boldsymbol{x})$ defined by Equation can be considered a result of uncertainty propagation. The "lower-level" aleatory uncertainties in characteristics of the initiating event $E_{0}$ are transformed into the "higher-level" aleatory uncertainties in the components of $\boldsymbol{x}$. The "lower-level" uncertainties are quantified by cdf $F_{0}\left(z_{0} \mid \theta_{0}\right)$, whereas the "higher-level" uncertainties are expressed by the family of empirical cdf's $\hat{F}_{i}(\boldsymbol{x})(i=1,2,3)$. At the same time, the "lower-level" epistemic uncertainties related to the models $M_{0}\left(z_{0} \mid \boldsymbol{\theta}_{0}, p_{0}\right), \boldsymbol{m}_{1}\left(z_{1} \mid \pi_{1}\right), m_{2}\left(z_{2} \mid \pi_{2}\right)$, and $\boldsymbol{m}_{3}\left(z_{3} \mid \pi_{3}\right)$ are transformed into the "higher-level" distributions $p_{i}(i=1,2,3)$ and $F_{P_{a}}\left(p_{a} \mid \theta_{P_{a}}\right)$.

This case study served only illustrative purposes. Therefore input information expressed by pd's related to $M_{0}\left(z_{0} \mid \theta_{0}, p_{0}\right), \quad \boldsymbol{m}_{1}\left(z_{1} \mid \pi_{1}\right)$, $m_{2}\left(z_{2} \mid \pi_{2}\right)$, and $\boldsymbol{m}_{3}\left(z_{3} \mid \pi_{3}\right)$ is hypothetical. In practice, the selection of the pam $F r_{X}(\boldsymbol{x})$ would require collecting hard data and eliciting expert judgements. On the other hand, the present case study may be considered to be a useful intermediate result. It provides a list of physical variables and model parameters for which initial information is to be obtained in order to specify probability distributions used as input in the problem of selecting $\mathrm{Fr}_{X}(\boldsymbol{x})$.

\section{Conclusions}

This paper illustrated an application of the simulation-based procedure developed for forecasting mechanical effects of accidental explosions on the road [1]. The form of this forecasting was a probabilistic model selected for actions induced by an accidental explosion (AE). It was suggested to fit the model to the multi-dimensional statistical samples generated during a stochastic simulation of accident involving AE. This simulation was applied to propagating of uncertainties in the physical phenomena leading to an occurrence of AE.

The simulation was based on the classical Bayesian approach to the quantitative risk assessment. Its final result (the probabilistic action model) was formulated in line with this approach and expressed the aleatory and epistemic uncertainties related to characteristics of the incident blast wave generated by AE.

The main message which can be concluded from the case study described above is that a relatively large amount of knowledge is necessary for selecting probabilistic action model for AE. In addition, subjective information (expert opinions) may play a substantial role in input information used for the accident simulation. The classical Bayesian approach provides mathematical means for expressing this information in the form of prior (posterior) probability distributions. The case study gave recipes how to handle them in light of predicting actions induced by AEs. It was found that the number of prior distributions to be specified is not too high.

An intermediate result of modelling the road accident involving $\mathrm{AE}$ was a large number of statistical samples generated by means of the stochastic simulation. A classification of these samples was applied to select the action model in the form of a family of probability distributions expressing both aleatory and epistemic uncertainty. This model can be updated if new information becomes available. The number of simulations (Monte Carlo trials) used to generate the aforementioned samples was relatively low.

In view of the structural engineering, the model describing effects of AEs is an intermediate result characterising the incident blast wave. To carry out the assessment of damage from AE, this model must be transformed into one which describes blast wave reflected by the specific exposed building. However, such a transformation is not directly related to the problem of transportation of hazardous materials. Therefore it was beyond the scope of the case study presented in the second part of this paper. 


\section{References}

1. Vaidogas, E. R. Simulation-based forecasting effects of an accidental explosion on the road. Part I: Methodological framework. Transport, Vol XXI, No 3, 2006, p. 165-171.

2. Wharton, R. K. et al. Airblast TNT equivalence for a range of commercial blasting explosives. Journal Hazardous Materials, Vol A79, 2000, p. 31-39.

3. Bulson, P. S. Explosive loading on engineering structures. London etc: E \& FN Spon, 1997. 236 p.

4. Baker, W. E. et al. Explosion hazards and valuation. Amsterdam etc: Elsevier, 1983. 432 p.

5. Kumamoto, H.; Henley, E. J. Probabilistic risk assessment for engineers and scientists, 2nd ed. New York: IEEE Press, 1996. 511 p.

6. Aven, T.; Pörn, K. Expressing and interpreting the results of quantitative risk analyses. Review and discussion. Reliability Engineering \& System Safety, Vol 61, 1998, p. 3-10.

7. Noon, R. K. Engineering analysis of vehicular accidents. Boca Raton: CRC Press, 1994. 239 p.

8. Ivanov, K.; Lialin, V. A. Passive safety of car. Moscow: Transport, 1979. 233 p. (in Russian).

9. Luzin, O. V. et al. Analysis of blast-loaded structures. In: Korenev, B., editor. Dynamics of structures exposed to special effects. Moscow: Stroiizdat, 1982, p. 5-28 (in Russian).

10. Tedesco, J. W.; McDougal, W. G.; Ross, C. A. Structural dynamics. Theory and applications. Menlo Park, California: Addison Wesley, Longman, Inc., 1999. 714 p.

11. Vaidogas, E. R. Actions imposed on structures during man-made accidents: Prediction via simulation-based uncertainty propagation. Journal of Civil Engineering and Management, Vol XI, No 3, 2005, p. 225-242.

12. Vaidogas, E. R. Procedure for sample classification with the aim of quantifying epistemic uncertainties in fitted probabilistic models. In: Manstaviciene Z., editor. Abstracts of Communications, 8th Vilnius Conf. on Probability Theory and Mathematical Statistics. Vilnius: TEV, 2002, p. 329-330.

13. Siu, N.O.; Kelly, D. Bayesian parameter estimation in probabilistic risk assessment. Reliability Engineering \& System Safety, Vol 62, 1998, p. 89-116.

14. Congdon, P. Bayesian statistical modelling. Wiley, Chischester etc., 2001.354 p.

15. Mosleh, A.; Apostolakis, G. The assessment of probability distributions from expert opinions with an application to seismic fragility curves. Risk Analysis, Vol 6, 1986, p. $447-461$.

\section{Appendix. Assigning and updating the epistemic uncertainty distributions used in the case study}

\begin{tabular}{l} 
Epistemic cdf $F_{P_{0}}\left(p_{0} \mid \theta_{P_{0}}\right)$ of parameters $\theta_{P_{0}}$ of $M_{0}\left(z_{0} \mid \theta_{0}, p_{0}\right)$ \\
\hline New data: $E=\{r$ collisions in the time interval $[0, t]\}$ \\
Updating prior: for the alpha parameter, $\theta_{1 P_{0}}^{\prime}=\theta_{1 P_{0}}+r$; for the beta parameter, $\theta_{2 P_{0}}^{\prime}=\theta_{2 P_{0}}+t$ \\
Constructing prior: constructing the prior distribution for $\theta_{P_{0}}$ is considered e.g. in $[13]$ \\
Epistemic cdf $F_{\theta_{60}}\left(\theta_{60}\right)$ of parameters $\theta_{60}$ of cdf $F_{Z_{60}}\left(z_{60} \mid \theta_{60}\right)$ \\
\hline New data: $E=\left\{z_{60, i}, i=1,2, \ldots\right\}$, where $z_{60, i}=$ value of $Z_{60}$ obtained from the $i$ th experiment (collision) \\
Updating prior: posterior distributions must be evaluated numerically using the Bayes formula for density updating \\
Constructing prior: constraints on the distribution moments, $0<\theta_{1,60}<1$ and $0<\theta_{2,60}<0.25$, allow to apply the maximum-entropy \\
method to assigning the prior density $f_{\theta_{60}}\left(\theta_{60}\right)[13]$
\end{tabular}

\footnotetext{
Epistemic c..d.f. $F_{\theta_{2}}\left(\theta_{2}\right)$ of parameters components of $\theta_{2}$ of $\operatorname{cdf} F_{22}\left(z_{2} \mid \theta_{2}\right)$

New data: $E=\left\{\left(z_{2 i}, P_{e}\left(E_{2} \mid z_{2 i}\right)\right), i=1,2, \ldots\right\}$, where $z_{2 i}=$ value of the collision energy $z_{2} ; P_{e}\left(E_{2} \mid z_{2 i}\right)=$ estimate of the probability of explosion $E_{2}$ given a collision with the energy $z_{2 i}$

Updating prior: assigning and updating cdf $F_{\theta_{2}}\left(\theta_{2}\right)$ can be stated as a problem of a simple linear regression analysis in the Bayesian setting if the pairs $\left(z_{2 i}, P_{e}\left(E_{2} \mid z_{2 i}\right)\right), i=1,2, \ldots$ are represented in the coordinate system of a normal probability graph paper; the components of $\theta_{2}$ can be expressed as functions of uncertain linear regression parameters and the prior $F_{\Theta_{2}}\left(\theta_{2}\right)$ improved by updating these regression parameters, see e.g. [14] for updating priors of regression parameters Constructing prior: see [14] for assigning priors to parameters of linear regression models; see also [15]for estimating fragility functions from expert opinions

$$
\text { Epistemic cdf's } F_{\Theta_{i 1}}\left(\theta_{i 1} \mid \theta_{\Theta_{i 1}}\right)(i=1,2), F_{\theta_{i 3}}\left(\theta_{i 3} \mid \theta_{\theta_{i 3}}\right)(i=1,2,3,4) \text { of the respective parameters }
$$$$
\theta_{11}, \theta_{21}, \theta_{13}, \theta_{23}, \theta_{33}, \theta_{43} \text { of } \boldsymbol{m}_{1}\left(z_{1} \mid \theta_{1}\right) \text { and } \boldsymbol{m}_{3}\left(z_{3} \mid \theta_{3}\right)
$$

New data: $E^{\prime}=\left\{\pi_{i}^{\prime}, i=1,2, \ldots\right\}, E^{\prime \prime}=\left\{\ln \pi_{i}^{\prime \prime}, i=1,2, \ldots\right\}, E^{\prime \prime \prime}=\left\{\ln \pi_{i}^{\prime \prime \prime}, i=1,2, \ldots\right\}$, where $\pi_{i}^{\prime}=$ experimental value of the tolerable energy of collision; $\pi_{i}^{\prime \prime}$ and $\pi_{i}^{\prime \prime \prime}=$ experimental values of relative overpressure and relative impulse Updating prior: procedures developed for updating priors of mean and variance (precision) of a normal pd allow expressing posterior distributions in closed form, see e.g. [14]

Constructing prior: see prior constructing procedures given in [14]

Epistemic cdf's $F_{\theta_{i 3}}\left(\theta_{i 3} \mid \theta_{\theta_{i 3}}\right)(i=5,6,7,8)$ of the respective parameters $\pi_{53}, \pi_{63}, \pi_{73}, \pi_{83}$ of $\boldsymbol{m}_{3}\left(z_{3} \mid \theta_{3}\right)$

New data: $E^{\prime}=\left\{\left(p_{i}^{+}, z_{13, \mathrm{i}}, z_{23, \mathrm{i}}\right), i=1,2, \ldots\right\}, E^{\prime \prime}=\left\{\left(l_{i}^{+}, z_{13, i}, z_{23, i}\right), i=1,2, \ldots\right\}$, where $p_{i}^{+}$and $l_{i}^{+}=$values of the positive overpressure and positive impulse measured in the ith experiment, respectively; $z_{13, i}$ and $z_{23, i}=$ mass and standoff of explosive charge used in the $i$ th experiment, respectively

Updating prior: $\theta_{53}, \theta_{63}, \theta_{73}$, and $\theta_{83}$ are parameters of the nonlinear, multiple regression models $m_{13}^{\prime}\left(z_{3} \mid \cdot\right)$ and $m_{23}^{\prime}\left(z_{3} \mid \cdot\right)$; to the best of our knowledge posterior distributions of these parameters can not be expressed in closed form and, moreover, practical procedures of numerical updating priors of $\theta_{53}, \theta_{63}, \theta_{73}$, and $\theta_{83}$ specifically and parameters of nonlinear regression models generally are still to be developed

Constructing prior: the cdf's $F_{\theta_{i 3}}\left(\theta_{i 3} \mid \theta_{\theta_{i 3}}\right)(i=5,6,7,8)$ were chosen by assigning normal distributions to respective regression parameters; mean values of these distributions were chosen to be equal to values of conventional least squares estimates of $\theta_{53}, \theta_{63}, \theta_{73}$, and $\theta_{83}$ given in [9]
} 